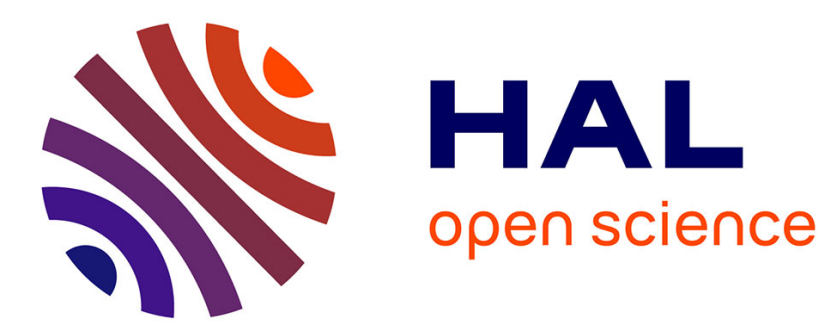

\title{
Courants thermostimulés et thermoluminescence dans AgGaS2
}

\author{
J.P. Leyris, J.P. Aicardi, S. Soule
}

\section{To cite this version:}

J.P. Leyris, J.P. Aicardi, S. Soule. Courants thermostimulés et thermoluminescence dans AgGaS2. Revue de Physique Appliquée, 1983, 18 (3), pp.147-152. 10.1051/rphysap:01983001803014700 . jpa00245079

\section{HAL Id: jpa-00245079 https://hal.science/jpa-00245079}

Submitted on 1 Jan 1983

HAL is a multi-disciplinary open access archive for the deposit and dissemination of scientific research documents, whether they are published or not. The documents may come from teaching and research institutions in France or abroad, or from public or private research centers.
L'archive ouverte pluridisciplinaire HAL, est destinée au dépôt et à la diffusion de documents scientifiques de niveau recherche, publiés ou non, émanant des établissements d'enseignement et de recherche français ou étrangers, des laboratoires publics ou privés. 


\title{
Courants thermostimulés et thermoluminescence dans $\mathbf{A g G a S}_{2}$
}

\author{
J. P. Leyris, J. P. Aicardi et S. Soule \\ Laboratoire de Physique du Solide, Université de Perpignan, \\ Avenue de Villeneuve, 66025 Perpignan Cedex, France
}

(Reçu le 15 septembre 1982, révisé le ler décembre, accepté le 15 décembre 1982)

\begin{abstract}
Résumé. - Des mesures de courants thermostimulés (TSC) et de thermoluminescence (TL) ont été effectuées sur des cristaux de $\mathrm{AgGaS}_{2}$ (de haute résistivité) entre 10 et $270 \mathrm{~K}$. Aux environs de $40 \mathrm{~K}$ le spectre montre simultanément un pic de TSC et de TL. La corrélation entre ces deux phénomènes nous permet d'expliquer le processus de recombinaison. Dans le domaine compris entre 70 et $200 \mathrm{~K}$ nous observons plusieurs pics de TSC non résolus qui obéissent à une cinétique monomoléculaire. A plus haute température, les spectres de TSC sont dominés par deux niveaux à cinétique plus complexe. Le calcul montre que la profondeur des niveaux mis en jeu est similaire à celle obtenue dans nos études de luminescence.

Abstract. - Thermally stimulated current (TSC) and thermoluminescence (TL) measurements are performed in high resistivity $\mathrm{AgGaS}_{2}$ crystals between 10 and $270 \mathrm{~K}$. About $40 \mathrm{~K}$ the spectrum shows at the same time TSC and TL peaks. Correlation between this two phenomena permits us to explain the recombination process. In the range 70 to $200 \mathrm{~K}$ we observed several overlapping TSC's peaks which obey to monomolecular kinetic. At higher temperature the TSC spectra are dominated by two levels with complex kinetic. The levels depth calculated are similar to those obtained in our luminescence studies.
\end{abstract}

1. Introduction. - Le thiogallate d'argent $\left(\mathrm{AgGaS}_{2}\right)$ est un composé I-III-VI ${ }_{2}$ qui cristallise dans la structure chalcopyrite [1]. C'est un matériau intéressant en optique non linéaire [2-4] (domaine de transparence élevé de 0,15 à $12 \mu \mathrm{m}$ et de dichroïsme important). Des mesures d'absorption, de réflectivité modulée et d'électro-réflectance $[5,6]$ ont montré que son gap est direct $(E \mathrm{~g}=2,727 \mathrm{eV}$ à $2 \mathrm{~K})$. Il existe un certain nombre de travaux concernant les propriétés optiques de ce matériau, par contre ses propriétés électriques sont mal connues. Il est difficile d'étudier les phénomènes de transport électrique sur ce composé car il est très isolant $\left(R \simeq 10^{9} \Omega \mathrm{cm}\right)$ et fortement compensé $[5,7,8]$. Des mesures d'effet Hall ont montré que les cristaux "as grown" sont de type $\mathbf{P}$, la mobilité est très faible (de l'ordre de $20 \mathrm{~cm}^{2} / \mathrm{Vs}$ ). Cet article présente des mesures de courant thermostimulé et de thermoluminescence, la technique de processus thermostimulés étant bien adaptée pour ce type de matériau très compensé. Cette étude a pour but de compléter celles que nous avions entreprises en luminescence $[9,10]$ et de permettre de mieux caractériser les propriétés physiques des niveaux pièges mis en jeu (section de capture, cinétique de la recombinaison, énergie d'activation).
2. Technique expérimentale. - Les cristaux de thiogallate d'argent que nous avons utilisés ont été fabriqués suivant la méthode Bridgman par $\mathbf{H}$. Matthes [11]. Les échantillons se présentent sous la forme de plaquette d'épaisseur $L=0,7 \mathrm{~mm}$ et de surface $S=6 \mathrm{~mm}^{2}$. Les électrodes semi-transparentes sont obtenues par évaporation sous vide d'un mince film d'or $(\simeq 200 \AA)$.

La tension de polarisation appliquée à l'échantillon est de $40 \mathrm{~V}$. Des mesures d'injection nous montrent qu'à ce voltage nous pouvons considérer les contacts comme ohmiques. L'excitation optique se fait avec une lampe à vapeur de zénon de $450 \mathrm{~W}$ suivie d'un monochromateur qui permet de sélectionner l'énergie d'excitation.

L'échantillon est refroidi par un cryostat à récupération de chaleur sensible (type Air Liquide CRCS) couplé à un régulateur de température. Le domaine de variation en température s'étend de $4,2 \mathrm{~K}$ à $300 \mathrm{~K}$, la vitesse de chauffe est linéaire (elle peut varier de 0,017 à $0,250 \mathrm{~K} / \mathrm{s}$ ) la régulation est assurée à $0,1 \mathrm{~K}$.

3. Résultats expérimentaux. - 3.1 AlluRE GÉNÉRALE DES SPECTRES. - Nous montrons sur la figure 1 l'allure générale des courbes de courant thermo- 


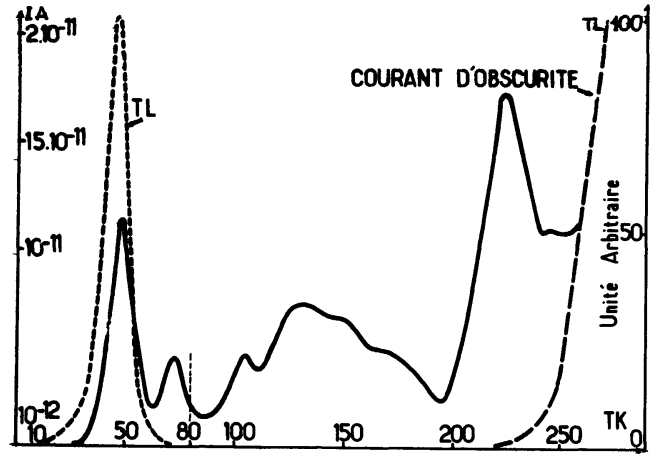

Fig. 1. - Allure générale du spectre de TSC et de TL : -TSC; -.- TL.

[General shape of TSC and TL spectra :- $—$ TSC ; -.- TL.]

stimulées (TSC) et de thermoluminescence (TL) dans l'intervalle de température de $10 \mathrm{~K}$ à $2^{\prime} / 0 \mathrm{~K}$. Ces spectres sont obtenus pour une durée d'excitation de $30 \mathrm{~min}$ à $T=7 \mathrm{~K}$, pour une longueur d'onde d'excitation de $\lambda=4700 \AA$ et une vitesse de chauffe de $\beta=0,166 \mathrm{~K} / \mathrm{s}$.

Plusieurs pics apparaissent aux températures $T_{\mathrm{m}}=48,73,105,131,150,166,224$ et $235 \mathrm{~K}$.

Nous avons fait des mesures en fonction du temps d'excitation :

Dans le domaine de température entre $70 \mathrm{~K}$ et $200 \mathrm{~K}$, la position des maxima de TSC ne change pas avec la durée d'excitation. On observe :

- Pour les temps d'excitation les plus courts (entre $10 \mathrm{~s}$ et $2 \mathrm{~min}$ ) un maximum à $166 \mathrm{~K}$ et des épaulements à 150 et $105 \mathrm{~K}$.

- Pour les temps d'excitation compris entre 5 min et $1 \mathrm{~h}$, un extremum à $150 \mathrm{~K}$ et des épaulements à 105 , 130 et $166 \mathrm{~K}$.

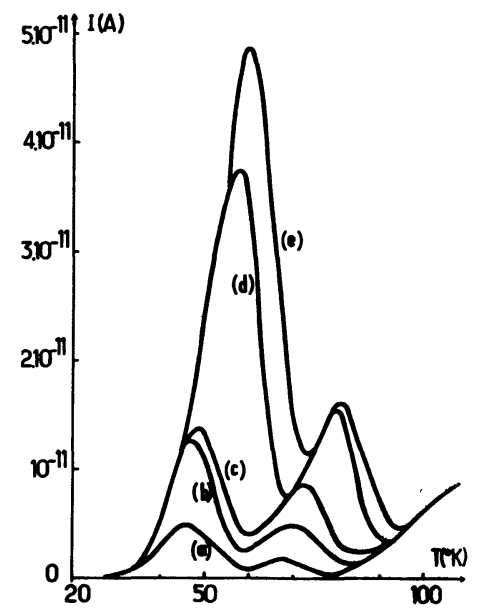

Fig. 2. - Forme des spectres de TSC en fonction de la vitesse de chauffe $\beta$ (échantillon excité à $20 \mathrm{~K})[(a) 5 \mathrm{~K} / \mathrm{min}$, (b) $10 \mathrm{~K} / \mathrm{min}$, (c) $20 \mathrm{~K} / \mathrm{min}$, (d) $25 \mathrm{~K} / \mathrm{min}$, (e) $30 \mathrm{~K} / \mathrm{min}$ ].

[Shape of the TSC curves as a function of the heating rate (sample excited at $20 \mathrm{~K}$ ) [(a) $5 \mathrm{~K} / \mathrm{min},(b) 10 \mathrm{~K} / \mathrm{min}$, (c) $20 \mathrm{~K} / \mathrm{min}$, (d) $25 \mathrm{~K} / \mathrm{min},(e) 30 \mathrm{~K} / \mathrm{min}]$.]

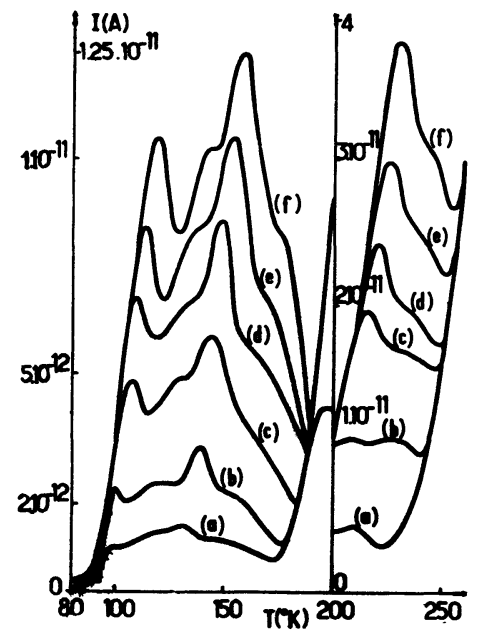

Fig. 3. - Forme des spectres de TSC en fonction de la vitesse de chauffe $\beta$ (échantillon excité à $80 \mathrm{~K}$ ) $[(a) 2 \mathrm{~K} / \mathrm{min}$, (b) $5 \mathrm{~K} / \mathrm{min}$, (c) $10 \mathrm{~K} / \mathrm{min}$, (d) $15 \mathrm{~K} / \mathrm{min}$, (e) $20 \mathrm{~K} / \mathrm{min}$, (f) $25 \mathrm{~K} / \mathrm{min}$ ].

[Shape of the TSC curves as a function of the heating rate (sample excited at $80 \mathrm{~K}$ ) $[(a) 2 \mathrm{~K} / \mathrm{min}$, (b) $5 \mathrm{~K} / \mathrm{min}$, (c) $10 \mathrm{~K} / \mathrm{min}$, (d) $15 \mathrm{~K} / \mathrm{min}$, (e) $20 \mathrm{~K} / \mathrm{min}$, (f) $25 \mathrm{~K} / \mathrm{min}$ ].]

- Au-dessus de $200 \mathrm{~K}$, un maximum aux environs de $220 \mathrm{~K}$, qui se déplace vers les basses températures quand le temps d'excitation augmente.

L'évolution des spectres en fonction de la vitesse de chauffe constitue une des méthodes d'exploitation des processus thermostimulés. Les figures 2 et 3 représentent les courbes de TSC pour différentes vitesses de chauffe. Nous observons un déplacement des spectres vers les hautes températures quand la vitesse de chauffe augmente.

3.2 ANAlyse Des Résultats. - Nous avons divisé cette analyse en trois parties :

- Le domaine de température s'étendant entre $70 \mathrm{~K}$ et $200 \mathrm{~K}$ où nous observons que la position en température des maxima ne se déplace pas quand la densité d'excitation varie. Ceci nous permet de dire que le processus de la recombinaison est du type monomoléculaire.

- Au-dessus de $200 \mathrm{~K}$, jusqu'à la température ambiante, la position des pics varie avec l'excitation, preuve que la cinétique de la recombinaison est probablement plus complexe.

- Nous terminerons enfin notre étude par un niveau piège situé près des bandes de transport. La relaxation de ce piège qui se produit à basse température (entre $40 \mathrm{~K}$ et $50 \mathrm{~K}$ ) se caractérise expérimentalement par l'observation simultanée d'un pic de courant stimulé et de thermoluminescence.

3.2.1 Zone de température comprise entre $70 \mathrm{~K}$ et $200 \mathrm{~K}$. - Dans cette région nous avons utilisé plusieurs méthodes classiques afin de déterminer les paramètres des courbes de TSC. Elles s'appuient soit sur l'évolution des spectres avec la vitesse de 
chauffe, soit sur l'examen direct de la forme des courbes. La méthode d'Hoogenstraten [12] est basée sur la variation du maximum $T_{\mathrm{m}}$ des courbes en fonction du taux de rechauffage $\beta$.

On obtient :

$$
\ln \frac{T_{\mathrm{m}}^{2}}{\beta}=\frac{E_{\mathrm{t}}}{k T_{\mathrm{m}}}-\ln \frac{k v}{E_{\mathrm{t}}},
$$

$E_{\mathrm{t}}=$ profondeur du niveau mis en jeu.

Pour un repiégeage faible, la fréquence d'échappement est :

$$
v=N_{\mathrm{c}, \mathrm{v}} S_{\mathrm{t}} V_{\mathrm{th}}
$$

avec

$N_{\mathrm{c}, \mathrm{v}}=$ densité d'équivalence d'état de la bande de conduction (valence),

$S_{\mathrm{t}}=$ section efficace de capture du piège,

$V_{\mathrm{th}}=$ vitesse thermique.

Si l'on trace $\ln \frac{T_{\mathrm{m}}^{2}}{\beta}$ en fonction de $1 / T_{\mathrm{m}}$, on obtient une droite dont la pente fournit la valeur de $E_{\mathrm{t}}$ et l'ordonnée à l'origine $v$, qui nous permet d'en déduire la section efficace de capture du piège.

Haering-Adams [13] exploitent la conductivité au maximum $\sigma_{m}$ à différentes vitesses de chauffe.

$$
\ln \sigma_{\mathrm{m}}=-\frac{E_{\mathrm{t}}}{k T_{\mathrm{m}}}-\left(1-\ln \sigma_{0}\right),
$$

avec

$\sigma_{0}=e \mu \tau n_{\mathrm{t}_{0}} v$ pour un repiégeage faible,

$\mu=$ mobilité,

$\tau=$ durée de vie de la recombinaison,

$n_{t_{0}}=$ nombre de porteurs piégés à basse température.

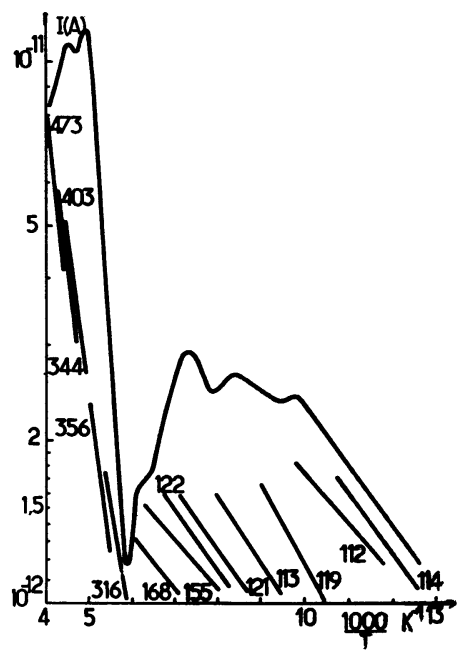

Fig. 4. - Courbe de vidage fractionné entre $80 \mathrm{~K}$ et $270 \mathrm{~K}$ (énergie en $\mathrm{meV}$ ).

[Fractional trap emptying curves between $80 \mathrm{~K}$ and $270 \mathrm{~K}$ (energy in $\mathrm{meV})$.]

La méthode de Garlick-Gibson [14] est applicable quel que soit le type de cinétique. Elle utilise la pente

\begin{tabular}{|c|c|c|c|c|c|c|}
\hline \multicolumn{2}{|c|}{$\begin{array}{l}\text { Méthode } \\
\text { d'analyse } \\
T_{\mathrm{m}}\end{array}$} & Hoogenstraten & Haering-Adams & Garlick-Gibson & Halperin-Braner & Vidage fractionné \\
\hline \multirow{2}{*}{$73 \mathrm{~K}$} & $E_{\mathrm{t}}(\mathrm{meV})$ & 68 & 71 & 66 & 62 & \\
\hline & $S_{\mathrm{t}} \mathrm{cm}^{2}$ & $1,02 \times 10^{-21}$ & & & & \\
\hline \multirow{2}{*}{$105 \mathrm{~K}$} & $E_{\mathrm{t}}(\mathrm{meV})$ & 115 & 110 & 113 & & 115 \\
\hline & $S_{\mathrm{t}} \mathrm{cm}^{2}$ & $2,66 \times 10^{-21}$ & & & & \\
\hline \multirow{2}{*}{$131 \mathrm{~K}$} & $E_{\mathrm{t}}(\mathrm{meV})$ & & & & & 121 \\
\hline & $S_{\mathrm{t}} \mathrm{cm}^{2}$ & & & & & \\
\hline \multirow{2}{*}{$150 \mathrm{~K}$} & $E_{\mathrm{t}}(\mathrm{meV})$ & 121 & 125 & & & 122 \\
\hline & $S_{\mathrm{t}} \mathrm{cm}^{2}$ & $3,76 \times 10^{-23}$ & & & & \\
\hline \multirow{2}{*}{$166 \mathrm{~K}$} & $E_{\mathrm{t}}(\mathrm{meV})$ & 162 & 172 & 195 & & 150 \\
\hline & $S_{\mathrm{t}} \mathrm{cm}^{2}$ & $2,2 \times 10^{-22}$ & & & & \\
\hline
\end{tabular}

Tableau I. - Paramètres des pics de cinétique monomoléculaire.

[Parameters of the monomolecular kinetic peaks.] 
initiale des courbès à basse température où $\sigma$ est donné par :

$$
\sigma=\sigma_{0} \exp \left(-E_{\mathrm{t}} / k T\right) .
$$

Dans le cas où nos pics de TSC sont mal résolus, nous avons utilisé la méthode du vidage fractionné [15]. Cette technique permet à l'aide de cycles successifs de rechauffage et de refroidissement de l'échantillon de déterminer l'énergie d'activation $E_{\mathrm{t}}$ des pièges.

Sur la figure 4, nous traçons ces courbes de vidage fractionné de $77 \mathrm{~K}$ à $250 \mathrm{~K}$.

Les méthodes basées sur la géométrie de la courbe nécessitent d'avoir un spectre bien isolé où l'on peut mesurer précisément les températures à mi-hauteur et au maximum.

Les formules utilisées sont très nombreuses et leurs applications nécessitent certaines conditions [16]. Nous nous limitons à l'expression donnée par Halperin et Braner [17] où la condition imposée est satisfaite dans notre cas.

$$
E_{\mathrm{t}}=\frac{K T_{\mathrm{m}}^{2}}{T^{\prime \prime}-T_{\mathrm{m}}}
$$

avec $T^{\prime \prime}$ et $T^{\prime}$ températures à mi-hauteur $T^{\prime}<T_{\mathrm{m}}<T^{\prime \prime}$, et la condition

$$
\frac{T^{\prime \prime}-T_{\mathrm{m}}}{T^{\prime \prime}-T^{\prime}} \simeq 0,37
$$

Ces différentes méthodes nous ont permis de calculer les énergies d'activation et les sections efficaces de capture des pics à $T_{\mathrm{m}}=73,105,131,150$ et $166 \mathrm{~K}$. Le tableau I résume ces résultats. Notons que l'énergie d'activation du pic situé à $131 \mathrm{~K}$ est imprécise, ce maximum n'étant jamais résolu.

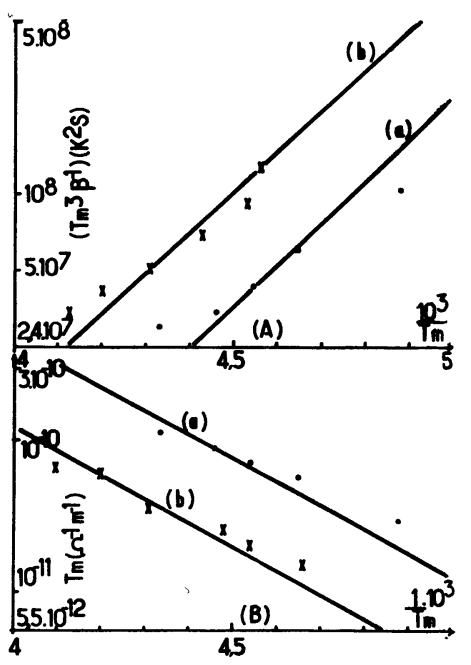

Fig. 5. - Pic à $224 \mathrm{~K}$. Droites d'Hoogenstraten (A) et d'Haering-Adams (B) à deux temps d'excitations : (a) $1 \mathrm{~h}$, (b) $10 \mathrm{~s}$.

[224 K peak. Hoogenstraten's (A) and Haering-Adams' (B) for two excitation times : (a) $1 \mathrm{~h},(b) 10 \mathrm{~s}$.]
3.2.2 Domaine de température au-dessus de $200 \mathrm{~K}$. - Le pic que nous observons à $224 \mathrm{~K}$ se déplace vers les basses températures quand on augmente l'excitation. La cinétique à laquelle il obéit est du type bimoléculaire. Nous avons tracé sur la figure 5 les droites d'Hoogenstraten correspondant à ce processus de recombinaison pour deux excitations différentes. Quand on porte :

$$
\ln \frac{T_{\mathrm{m}}^{3}}{\beta}=\frac{E_{\mathrm{t}}}{k T_{\mathrm{m}}}+\ln \left(\frac{k E_{\mathrm{t}}^{2}}{2 n_{\mathrm{t}}} S_{\mathrm{r}} \frac{N_{\mathrm{c}}}{N_{\mathrm{t}}}\right),
$$

en fonction de $1 / K T_{\mathrm{m}}$ on obtient une droite de pente $E_{\mathrm{t}}$. L'ordonnée à l'origine dépend en particulier du taux de remplissage $n_{\mathrm{t}}$, de la section de capture du centre $S_{\mathrm{r}}$. Ceci explique que contrairement au cas monomoléculaire, on observe expérimentalement un glissement des droites lorsqu'on fait varier la densité d'excitation. Dans notre cas, les pentes de droites nous fournissent une énergie d'activation $E_{\mathrm{t}} \simeq 310 \mathrm{meV}$.

La pente initiale (Garlick-Gibson) et les vidages fractionnés donnent une profondeur du niveau de 330 à $350 \mathrm{meV}$.

Dans cette zone de température, on voit pour les temps d'excitation importants (de l'ordre de $2 \mathrm{~h}$ ) un pic de TSC aux environs de $235 \mathrm{~K}$. Le vidage fractionné (Fig. 4) fournit une énergie d'activation d'environ $440 \mathrm{meV}$.

3.2.3 Pics de TSC-TL aux très basses températures. - Nous avons exploité séparément chacun des deux processus (TSC et TL) en utilisant les méthodes développées précédemment. Nous résumons dans le tableau II les valeurs de la profondeur du niveau mis en jeu. Mais la comparaison de ces deux mécanismes simultanés constitue une information supplémentaire sur la connaissance du phénomène. En particulier, elle permet de préciser le type de la cinétique de recombinaison mise en jeu.

Dans notre cas, nous montrerons que ce dernier n'est pas monomoléculaire.

Tableau II. - Profondeur du niveau du pic basse température suivant les différentes méthodes d'analyse.

[Level depth of the low temperature peak by different methods.]

\begin{tabular}{|c|c|c|c|c|}
\hline $\begin{array}{c}\text { Méthode } \\
\text { d'analyse }\end{array}$ & $\begin{array}{c}\text { Hoogen- } \\
\text { straten }\end{array}$ & $\begin{array}{c}\text { Haering- } \\
\text { Adams }\end{array}$ & $\begin{array}{c}\text { Garlick- } \\
\text { Gibson }\end{array}$ & $\begin{array}{c}\text { Halperin- } \\
\text { Braner }\end{array}$ \\
\hline T.S.C. & $35 \mathrm{meV}$ & $50 \mathrm{meV}$ & $40 \mathrm{meV}$ & $30 \mathrm{meV}$ \\
\hline T.L. & $38 \mathrm{meV}$ & $55 \mathrm{meV}$ & $40 \mathrm{meV}$ & $36 \mathrm{meV}$ \\
\hline
\end{tabular}

J. P. Fillard et J. Gasiot $[18,19]$ ont développé une méthode originale basée sur la corrélation entre les TSC et les TL. Ils partent des expressions du courant 
et de l'intensité de la luminescence. Dans le cas d'une recombinaison libre-liée, on a :

$$
J_{\mathrm{TSC}}(T)=\mu(T) e E n_{\mathrm{c}}(T),
$$

pour le courant stimulé et

$$
J_{\mathrm{TL}}(T)=\frac{A n_{\mathrm{t}}(T)}{\tau(T)},
$$

pour la thermoluminescence ;

$n_{\mathrm{c}}(T)$ : nombre de porteurs libres,

$E \quad$ : champ appliqué,

$\tau(T)$ : durée de la recombinaison radiative.

L'étude du rapport $R=J_{\mathrm{TSC}} / J_{\mathrm{TL}}$ permet d'éliminer $n_{\mathrm{c}}(T)$.

Ce rapport ne dépend plus alors que du produit $\mu(T) \tau(T)$. Si $m(T)$ désigne la densité des centres recombinants encore libres à la température $T$, on pourra poser :

$$
\frac{1}{\tau(T)}=V_{\mathrm{th}} S_{\mathrm{r}}(T) m(T),
$$

$S_{\mathrm{r}}$ : section de capture du centre recombinant, et $R(T)$ s'écrit alors :

$$
R(T)=e E\left[\frac{\mu(T)}{V_{\mathrm{th}} S_{\mathrm{r}}(T)}\right] \frac{1}{m(T)}=e E \frac{f(T)}{m(T)},
$$

avec $f(T)=\frac{\mu(T)}{V_{\mathrm{th}} S_{\mathrm{r}}(T)}$.

Ce rapport $R(T)$ est un paramètre que l'on peut atteindre expérimentalement à partir des courbes de TSC et TL. Il dépend de la température par l'intermédiaire de $f(T)$ et de $m(T)$. Fillard et al. [18] suivent l'évolution de $f(T)$ dans leur domaine de température par une succession de vidage fractionné. Toutefois,

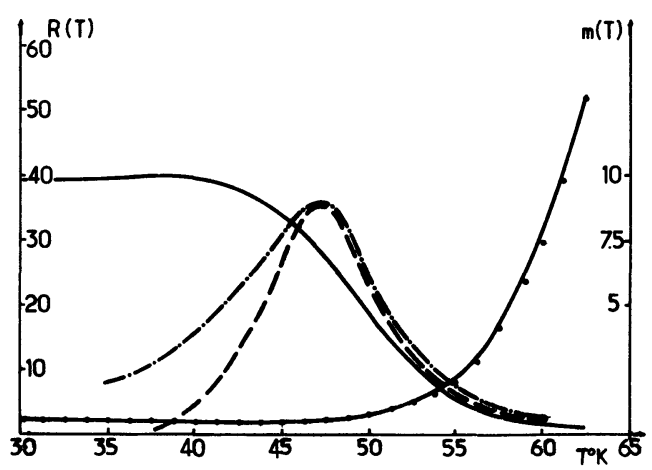

Fig. 6. - Etude du rapport $R(T)=J_{\mathrm{TSC}} / J_{\mathrm{TL}}$ pour le pic basse température. $\longrightarrow R(T),-\longrightarrow . J_{\mathrm{TL}}$, -.--- $\mathrm{d} m / \mathrm{d} T, \longrightarrow m(T)$.

[Study of the ratio $R(T)=J_{\mathrm{TSC}} / J_{\mathrm{TL}}$ for the low temperature peak. —. $R(T), \quad$ - —.—. $J_{\mathrm{TL}}, \quad \cdots \mathrm{d} m / \mathrm{d} T$ $m(T)$. dans notre cas, nos processus de relaxation se produisent entre $40 \mathrm{~K}$ et $55 \mathrm{~K}$ environ. On considérera que dans cet intervalle de température, la fonction $f(T)$ est constante. Dans le cas d'une recombinaison monoparticulaire $m(T)$ est constant et donc $R(T)$ doit rester constant. Pour une cinétique biparticulaire $m(T)$ varie avec la température. L'évolution de $R(T)$ est directement liée à celle de $m(T)$ et traduit l'évolution au cours du temps (car $T=b t+T_{0}$ ) du nombre de places sur le centre recombinant.

Nous portons sur la figure 6, l'allure expérimentale de $R(T)$ et celle de $m(T)$ qui en est déduite. Nous traçons aussi $\mathrm{d} m(T) / \mathrm{d} T$ qui est obtenue en dérivant la courbe $m(T)$. Nous avons également tracé sur la figure l'allure de la thermoluminescence.

On constate que $\mathrm{d} m(T) / \mathrm{d} T$ est identique à la courbe de TL. Ceci nous permet d'affirmer que la recombinaison radiative est le seul processus responsable de l'épuisement final du centre.

4. Discussion. - Nous pouvons comparer nos résultats à ceux donnés par H. J. Von Bardeleben et al. [20]. Ils obtiennent à une vitesse de chauffe de $0,8 \mathrm{~K} / \mathrm{s}$ une série de pics situés à $50,80,140,185$ et $214 \mathrm{~K}$. Seuls leurs maxima à $185 \mathrm{~K}$ et $214 \mathrm{~K}$ sont importants et bien résolus. Le premier est attribué à un niveau associé au nickel $\mathrm{Ni}^{3+}$, d'énergie d'activation d'environ $110 \mathrm{meV}$, le second à un piège à trous de $460 \mathrm{meV}$ de profondeur dû à un défaut lié au $\mathrm{Fe}^{3+}-\mathrm{X}$. La caractérisation chimique de leurs impuretés a été obtenue par des mesures de R.P.E.

Dans notre cas, toutefois, les pics observés sont mieux résolus et nous ont permis une exploitation plus complète des résultats. Notre optique a été de confirmer les niveaux que nous avions observés dans nos expériences de cathodoluminescence, ce qui a été possible dû au fait que nos résultats sont plus précis, et portent sur une plus grande quantité de maxima.

Les mesures à basse température ont été l'occasion de mettre en évidence une thermoluminescence qui n'avait jamais été signalée dans la littérature sur ce matériau. Cette transition que nous avons positionnée, à l'aide de filtres passe bande entre 0,83 et $1 \mu$, est à rapprocher d'une émission obtenue en cathodoluminescence et centrée à $1,362 \mathrm{eV}$. Elle correspond à une recombinaison de porteurs à partir d'une bande de transport sur un niveau situé au milieu de la bande interdite. Les énergies d'activation trouvées entre $4 \mathrm{~K}$ et $70 \mathrm{~K}$ ( 30 à $40 \mathrm{meV}$ d'une part, 60 à $75 \mathrm{meV}$ d'àutre part) sont à rapprocher des mesures de luminescence que nous avons faites au voisinage de la limite d'absorption [9] (J. P. Aicardi, J. P. Leyris, J. Phys. Chem. Solids, à paraître 82). Nous avions interprété les émissions observées à $2,678 \mathrm{eV}$ et $2,650 \mathrm{eV}$ comme des transitions du type libre-liée faisant intervenir un niveau donneur à $40 \mathrm{meV}$ et un niveau donneur (ou accepteur) aux environs de $75 \mathrm{meV}$ de la bande de transport. La série des pics non résolus compris entre $80 \mathrm{~K}$ et $190 \mathrm{~K}$ fournit des énergies d'activation entre 
110 et $160 \mathrm{meV}$. Le domaine de température où l'on observe ces maxima, ainsi que les énergies d'activation trouvées sont comparables à ceux du maximum à $185 \mathrm{~K}$ de $\mathrm{H}$. J. Von Bardeleben et al. Nos mesures confirment donc l'existence d'un niveau situé aux environs de $110 \mathrm{meV}$ de la bande de transport. Signalons que ce niveau d'impureté a été trouvé par P. W. Yu [7] à partir de mesures de résistivité en fonction de la température. Nous avions également montré qu'il était responsable d'une émission structurée au voisinage de la limite d'absorption, centrée à $2,593 \mathrm{eV}$.

Les pics à hautes températures sont ceux qui sont prédominants dans le spectre. Ils mettent en jeu des centres plus profonds. Le sommet à $235 \mathrm{~K}$ fournit une énergie d'activation (440 meV) du même ordre de grandeur que celle du pic à $214 \mathrm{~K}$ de [20].

En conclusion, nos études de TSC et TL à basse température nous ont permis de caractériser les paramètres et la cinétique des niveaux mis en jeu.

Il est évident, toutefois, que ces types de mesure (TSC et TL) sont toujours délicats à interpréter, en particulier à cause des nombreuses hypothèses simplificatrices qu'ils nécessitent pour l'interpretation des résultats. Dans notre cas, elles sont cependant intéressantes dans la mesure où l'on peut confirmer par des mesures électriques des niveaux d'impuretés observés par des expériences de luminescence, ce qui était l'objet de notre travail.

\section{Bibliographie}

[1] Shay, J. L. and WeRnick, J. H., Ternary Chalcopyrite Semi-Conductors (Pergamon Press) 1975.

[2] Matthes, H., Viehmann, R., Marschall, N. and KoRCZAK, P., Seconde Conférence Internationale sur les composés semi-conducteurs ternaires, $J$. Physique Colloq. 36 (1975) C3-105.

[3] Chemla, D. S., KupeCeK, P. J., Robertson, D. S. and SMith, R. C., Opt. Commun. 3 (1971) 29.

[4] Boyd, G. D., Kasper, H. and McFeE, I.E.E.E. J. Quantum Electron QE 7 (1971) 563.

[5] Tell, B., Kasper, H. M., Phys. Rev. B 4 (1971) 4455.

[6] Tell, B., Shay, J. L. and KasPer, H. M., Phys. Rev. B 6 (1972) 3008.

[7] Yu, P. W., Manthurnthil and Park, Y. S., J. Appl. Phys. 45 (1974) 3694.

[8] Yu, P. W. and Park, Y. S., J. Appl. Phys. 45 (1974) 823.

[9] Aicardi, J. P., Leyris, J. P. and Poure, A., J. Appl. Phys. 53 (1982) 1690.

[10] Aguero, G., Aicardi, J. P. et Leyris, J. P., J. Physique 42 (1981) 317.
[11] Matthes, H., ViehmanN, R. and Marschall, N., Appl. Phys. Lett. 26 (1975) 237.

[12] Hoogenstraten, W. P., Philips Res. Rep. 13 (1958) 515.

[13] Haering, R. R. and Adams, E. N., Phys. Rev. 2117 (1960) 451.

[14] Garlick, G. F. J. and Gibson, A. E., Proc. R. Soc. London, Ser. A 60 (1948) 574.

[15] Gobrecht, H. and Hofman, D. J., J. Phys. Chem. Solids 27 (1966) 509.

[16] Kivits, P. and HagebeUK, H. J. L., J. Lumin. 15 (1977) 1.

[17] Halperin, A. and Braner, A., Phys. Rev. 117 (1960) 408.

[18] Gasiot, J., Thèse (Montpellier) (1976).

[19] FillaRD, J. P., Thermally stimulated relaxation in solids (Springer Verlag Berlin Heidelberg New York) 1979, p. 35.

[20] Von Bardeleben, H. J., Schwab, C., Scharager, C., Muller, J. C., Siffert, P. and Feigelson, R. S., Phys. Status Solidi 58 (1980) 143. 